\title{
The protective scale of the Armidilo-S: the importance of forensic and clinical outcomes
}

\author{
William R. Lindsay \\ Lesley Steptoe \\ Fabian Haut \\ Sandra Miller \\ Jane Macer \\ Ronnie McVicker
}

This is the peer reviewed version of the following article:

Lindsay, W.R., Steptoe, L., Haut, F., Miller, S., Macer, J. \& McVicker, R. 2018. The protective scale of the Armidilo - S: the importance of forensic and clinical outcomes. Journal of Applied Research in Intellectual Disabilities.

which has been published in final form at doi:

http://dx.doi.org/10.1111/jar.12456

This article may be used for non-commercial purposes in accordance with the Wiley Terms and Conditions for SelfArchiving 
The Protective Scale of the Armidilo-S: The importance for accurate assessment and community safety

\author{
William R Lindsay ${ }^{\text {a }} \mathrm{c}$ \\ Lesley Steptoe ${ }^{\mathrm{cd}}$ \\ Sandra Miller ${ }^{\mathrm{c}}$ \\ Fabian Haut $^{\mathrm{c}}$ \\ Jane Macer ${ }^{b}$ \\ Ronnie McVicker ${ }^{\text {a }}$
}

${ }^{\text {a }}$ Danshell Healthcare, York, UK

${ }^{\mathrm{b}}$ University of West of Scotland, Glasgow, UK, Deakin Univ., Melbourne, Australia.

${ }^{\mathrm{c}}$ NHS Tayside, Dundee, UK

${ }^{\mathrm{d}}$ University of Abertay, Dundee

Keywords: Dynamic risk; intellectual disabilities; offenders; protective factors; ARMIDILO-S;

Correspondence: Dr Lesley Steptoe, Psychological Therapies, NHS Tayside, 1 Edward Street, Dundee, DD1 5NS, Scotland, UK, E-Mail lsteptoe@nhs.net 
Abstract

The Armidilo has two scales - the risk scale and the protective scale. Research has been confined to the risk scale which appears to predict future incidents with medium to large effect sizes. There have been no publications on the use of the protective scale.

The Armidilo was completed on 4 individuals with IDD who were either moving on from their placement or whose placement was in jeopardy because of new information or altered policies in the organisation. The Armidilo was completed in the usual fashion.

Risk and protective results show that for each individual, recommendations could be made that ensured the best outcome. For two participants restrictive placements were avoided because of the data on protective factors.

The protective scale can be a powerful support for the clinician's case in offenders with IDD. The protective scale should be completed routinely for clinical evaluation. 
Dynamic risk assessments contrast with static risk assessment in that the variables are able to be changed through treatment and management of the individual. Therefore, these variables are able to be incorporated into effective risk management plans for offenders with intellectual and developmental disabilities (IDD). Studies investigating the predictive value of dynamic variables with offenders with IDD have suggested that they may be as good as static variables in predicting future offences. Three early studies suggested the importance of clinical and dynamic indicators with this client group. Lindsay, Elliot and Astell (2004a) found that dynamic indicators of risk such as poor treatment compliance and anti-social attitudes, in 52 offenders with IDD, correlated with reoffending with a value as high or higher than static variables such as childhood behavioural and attachment problems. Quinsey, Book and Skilling (2004) in a study on 54 offenders with IDD found that in addition to the static variables on the VRAG, anti-social attitude (a dynamic indicator) also had a significant relationship with reoffending. McMillan, Hastings and Coldwell (2004) reported that a clinical rating of risk made by the multi-disciplinary team was as good a predictor of future violence as previous violent incidents.

Lindsay et al (2004b) and Steptoe et al (2008) developed the Dynamic Risk Assessment and Management System (DRAMS), including ratings of mood, anti-social behaviour, self-regulation, compliance and other clinical items. They found that the total score predicted violence over the next 2 days with an AUC of .73. This was a particularly powerful result since the occurrence or absence of the incident was so dynamic. In a field study with 30 offenders with IDD living in the community Lindsay et al (2017, in press) found that repeated assessments using the DRAMS predicted whether a participant was likely or not likely to perpetrate a violent incident in the following week with an AUC of 0.86 which is a very large effect size.

In relation to sexual incidents, Boer, Tough and Haaven (2004) developed the Assessment of Risk Manageability for Individuals with Developmental and Intellectual Limitations who Offend Sexually (Armidilo-S). The predictive value of this was assessed by Blacker et al (2011) in their study comparing 44 special needs with 44 mainstream offenders. With the special needs offenders, the Armidilo-S predicted incidents with AUCs between .73 and .76 and with the small sub group of 
offenders with ID $(\mathrm{n}=10)$ it predicted incidents with AUCs between .75 and .86 . In a study by Lofthouse et al (2013a) on 64 sex offenders with ID over a period of 6 years, predictive values for different sections of the ARMIDILO ranged between AUC .79 and .90 with an AUC of .92 for total score. These studies certainly suggest that the dynamic risk assessment may be a potent addition to risk management in this client group.

Because there seemed to be an emerging powerful relationship between dynamic risk factors and future incidents for this client group, Lofthouse et al (2012) reanalysed the risk assessment data on 212 offenders with IDD published by Lindsay et al (2008) where they had found that both the VRAG and the Short Dynamic Risk Scale (SDRS), an easily and quickly completed dynamic risk assessment, had equivalent risk predictive values of $\mathrm{AUC}=.71$ and .72 respectively. They used the model to investigate the functional relationships between risk factors developed by Kraemer et al (2001) which enables the investigator to determine whether the risk factors are overlapping, independent, mediating, moderating or acting as a proxy. They found that the dynamic variables on the SDRS acted as a proxy for the variables in the VRAG. They concluded that since these risk factors captured elements of the same underlying risk associated with violence, and since dynamic variables were more accessible and contributed towards risk management regimes, dynamic assessments could provide immediate information more relevant to intervention planning and the reduction of presenting risk.

These developments may have significant implications for the assessment and management of risk for people with IDD. The extent to which these studies can be replicated is extremely important for the future management of offenders with IDD. If it can be established that proximal indicators are not only more accessible, but also as predictive as static indicators, this may have a significant impact on procedures and practice in helping offenders with IDD to access services more adequately suited to the offender's current risk profile. Clinicians may have an awareness that there are protective factors, for example treatment effectiveness, that has positively influenced risk manageability however it can be difficult to argue this positive and dynamic aspect relative to the evidential static risk factors which may be subject to discussion within the risk management meeting. The crucial aspect is for further 
studies to be conducted in this area but to date there has been a dearth of publications regarding protective factors to be evidentially considered for offenders with IDD.

There is a slowly growing body of mainstream research investigating protective factors. The potential for assessments of protective factors to risk is demonstrated in reports on mainstream offenders. Willis and Grace (2008) found that social supports and clinical follow up were protective factors in the risk for future violent incidents in mainstream offenders. In the field of sex offenders with IDD, Lindsay et al (2002) reported that a planned follow up was a significant protective factor with a large effect size.

The Armidilo-S has become the principal dynamic risk assessment for sex offenders with IDD. It was also the first risk assessment to incorporate a protective scale (Boer et al, 2007). The protective scale is parallel but independent to the risk scale. For some items if the risk remains high e.g. high sexual preoccupation, but if the protective factor is also high e.g. constant supervision by experienced staff, the protective factor counters the risk factor. For other items the risk may have moderated e.g. high impulsivity lowering to moderate with corresponding improvements in the protective factor: low moderating to moderate impulse control. In this case the protective factor acts as the opposite of the risk, i.e. as the risk reduces the protection automatically increases. The crucial point is that the protective scale is independent, different factors will perform differently and the two scales are not added or subtracted in any way. They are considered separately for effect. In another example, the risk scale might be high with no protective rating e.g. poor supervision or staff unaware of sexual risk. Therefore, risk and protective scales can act in different ways to produce a final risk rating.

Two protective scales have been developed for mainstream offenders. The Structured Assessment of Protective Factors for violence risk (SAPROF; de Vogel et al 2012) was developed specifically to assess protective factors in relation to future violence. It contains three scales, each of which has specific items: the internal scale (e.g. intelligence; secure attachment; empathy); the motivational scale (e.g. work; leisure activities; motivation for treatment) and the external scale (e.g. social networks, intimate relationships, professional care). The SAPROF has been shown to be a good 
predictor of inpatient violence (Abidin et al 2013) and violent recidivism (de Vries Robbé, de Vogel, $\&$ de Spa, 2011). In an assessment of the usefulness of the SAPROF in combination with a risk assessment, de Vries Robbe et al (2016), found that it was a good predictor of institutional violence with 185 mentally disordered patients in a secure setting. Wilson et al (2010) assessed the protective scale of the Short-Term Assessment of Risk and Treatability (START; Webster et al 2009). The START has 20 items that result in estimates for personal vulnerabilities and strengths. Items include social, relationship, occupational and leisure skills, social supports and personal coping, insight and plans. After the initial assessment phase, they followed up 30 patients over one year. They found that the greater the participants' assessed strengths the less likely they were to engage in aggression over the follow up year. However, a strengths assessment did not contribute uniquely over the vulnerabilities scores to the predictions of aggression. These studies do, however, suggest that a consideration of client strengths and protective factors will be a very important area for future investigation in risk assessment.

This study is the first to investigate the utility of the protective scale for the ArmidiloS. The report concentrates on the effect that the scale has on clinical decision making and presents cases where the decisions have been directed or changed by the data on protective factors.

Method

Participants.

Four male sex offenders with IDD were chosen to illustrate the ways in which the protective scale of the Armidilo-S can be used. Their characteristics will be described in the results section. 


\section{Materials.}

The Static 99 (Hanson \& Thornton 2000). - (Hanson \& Thornton 2000). The Static99 is a well established actuarial instrument designed to measure long-term sexual recidivism. It contains 10 items covering four domains: persistence of sexual offending, sexual deviance, type of victims and anti-sociality. A raw total score is obtained (0-12), which is used to determine a recidivism risk estimate. Nominal risk categories can also be obtained from raw scores: '0' and ' 1 ' are categorized as Low Risk; '2' and '3' as MediumLow Risk; '4' and '5' as Medium-High Risk; and scores of '6' and above as High Risk. In the current study, the total score was used. The Static-99 has been shown to be an effective assessment for sexual offenders with IDD (Hanson, Sheahan \& VanZuylen 2013).

The Armidilo-S (Boer, Haaven, Lambrick, Lindsay, McVilly, Sakdalan \& Frize 2013)

- The Armidilo-S includes 26 items that have a dynamic relationship to risk or manageability of risk of intellectual disability sex offenders. The dynamic factors are defined as either stable (i.e. relatively enduring characteristics) or acute (i.e. rapidly changing contextual factors), and they refer to either the individual or the environment. The stable dynamic environmental items cover staff attitudes towards sex offenders with IDD, communication amongst supervisory staff, client-specific knowledge by staff, consistency of supervision including environmental consistency. Acute dynamic environmental items refer to any changes in supervisory staff, monitoring by staff, victim access and environmental changes such as relocation. Stable dynamic offender items involve compliance with supervision and treatment, offence management of risk factors and relapse prevention, sexual deviance and preoccupation, emotional coping, mental health, substance abuse, impulsive control, and relationships. Finally, the acute dynamic offender items refer to changes in supervision or treatment, changes in sexual preoccupation, changes in victim related behaviours and changes in emotional coping. Each of the four scales has a final item on individual or unique 
considerations for the person being assessed with examples in the manual for possible factors such as Autistic spectrum. Each item's presence over the previous period (2 years for stable items, 3 months for acute) is rated on a scale ranging from 0 to 2 , where $0=$ not present, $1=$ somewhat present, and 2 = definitely present. Lofthouse et al (2013a) found Cronbach's alphas were 0.71 for the total environment items, .86 for the total offender and 0.86 for the total Armidilo-S.

The protective scale reviews the same items with the same scoring system from 0 , not present as a protection, through 1 , somewhat present, to 2 , definitely present as a protection.

Procedure

The study received approval through the Caldecott system because all the data were already in the case files and were anonymised for publication. All the participants were assessed for clinical purposes using both the Armidilo and the Static 99. The information was then made available to interested parties for clinical purposes.

A convergent approach (Boer et al 2013) is recommended when using the Armidilo-S. This involves establishing a static risk assessment as a framework and then superimposing the findings from the Armidilo on to the static risk to determine the convergent risk for that individual. For each case a static 99 was conducted prior to administration of the Armidilo.

The administration of the Armidilo involved gathering data from three basic sources:

1. Observation and interview with the person themselves on each of the risk items.

2. Interviewing staff members for information about each item.

3. Case file review is also conducted to extract relevant information for each item.

Each item is then scored for a risk value and a protective value.

Results.

Case 1. Mr A. Mr A was aged mid-thirties and had a WAIS-IV UK IQ of 61. He had been charged and convicted of 7 sexual offences all of which occurred while drinking 
alcohol. All the offences were against adult women and ranged from voyeuristic behaviour to sexual assault. He was living in a group home for people with learning disabilities and had been in treatment for 2 years with a further 2 years regular follow up of at least monthly and often 2 weekly sessions. He had no reoffences and had not drunk alcohol for the four years of treatment. His current social worker was changed and the new social worker asked for an independent assessment of risk probability which suggested Mr A was a very high risk for future sexual offences based on the Static 99 and other static risk assessments. From the results of the risk assessment his social worker was of the opinion that the risks were too great to and asked for assessment for secure provision. An independent provider then assessed Mr A and found that he would be suitable for admission to a setting where both care provision and treatment could be provided in the community.

Figure 1 about here.

The issue for the residential establishment where he stayed and for us as treatment providers was that in our opinion Mr A had improved immeasurably both in terms of his drinking and his sexuality. He had improved on all post treatment measures related to offence related thinking (attitudes to women), alcohol use and emotional state however these more idiosyncratic protective factors were not reflected within the risk assessment process. The more dynamic protective factors in this case were therefore more difficult to evidence out with clinical opinion. The protective factor scale of the Armidilo S provides a constructive approach to the evaluation of protective factors which are relevant to risk probability. This report is on the effect of the protective scale of the Armidilo and demonstrate this within a figure to illustrate the way in which protective factors can be placed against risk factors.

Figure 1 shows some of the main risk factors that are assessed in the Armidilo. The results were reported in the presentation to both the social worker, the Multi Agency Public Protection Arrangements (MAPPA) and the low secure provider. The figure shows partial 
risk factors for poor impulse control, self-regulation of sexual preoccupation, mental health issues, alcohol misuse, emotional dysregulation, sexual coping and access to victims. This last point will always be at least a partial risk factor for anyone who lives in the community. The risk factors were evaluated conservatively so that there could be little argument against our risk assessment. All of the risk factors were set in a framework of high risk for future offending (Static 99).

The protective factors were then placed against the risk factors, always in the context of high risk for future sexual offending. The protective factors seen in fig 1 are good compliance with supervision (and treatment although this was not emphasised), no evidence of sexual deviance, excellent relationships with staff, well controlled mental health (through regular mental health monitoring), good alcohol regulation and agreement with a non alcohol regime in the residential home, somewhat effective emotional regulation (with regular psychiatric monitoring), good staff compliance, staff knowledge about Mr A's problems and stability within the staff group, partial access to victims and partially effective offence management regulation. We scored his sexual preoccupation as partially present although we had no good evidence but felt it cautious to assume it might still be present. In the face of possible sexual preoccupation (and no evidence to use anti libidinal medication) we had no protection against the item beyond staff monitoring. The complete Armidilo assessment can be seen in table 1. Perhaps the most interesting aspect is that there were no changes in the risk factors for any acute offender items and so to evaluate protective factors was viewed as having little utility at that time.

The discussion advanced through description of the protective factors that were set against the risk factors identified, which in turn, proved to make a persuasive case and it was decided, even in the context of accepting the high-risk Static 99 assessment, that the various parties were completely accepting of the case for community placement. There were further 
concerns when it was reported that Mr A had been staring at females in the community but, again, this was set against the protective factors in his Armidilo assessment and the placement was continued. Two years later he was placed in his own tenancy and a further three years on this placement continued to be successful.

Case 2. Mr B.

Table 1 about here.

Mr B was referred to residential placement following a series of incidents of indecent exposure and one incident of sexual assault. He was in his early twenties and assessment found him to have an IQ of 66 as measured by the WAIS IV UK. He had 5 charges of indecent exposure in shopping centres and two when he was in his garden. He also had three charges related to one incident of sexual assault against two females in a swimming pool. All the females were around his age from 19 to 26 and all the incidents were non-penetrative. The sexual assault charges were touching outside the clothing and one of attempting to put his hand down the female's waistband. None of the charges had been prosecuted because he was found unfit to plead.

Mr B was admitted to residential accommodation with very little background information and following admission he committed three further incidents of indecent exposure in the first few days. Mr B was already on MAPPA supervision and it was thought, following the further reports of sexual offences, that he should be considered for secure provision. A multi-agency case conference agreed to a period of four weeks to conduct an assessment of risk probability, start sex offender treatment to assess compliance, and assess him for the suitability of anti-libidinal medication. In fact there was little optimism that he could be managed in a community setting and it was expected that he would require secure provision to manage his sexuality. 
Assessment on the Static 99 placed him in the high-risk category for future sex offences with Mr B scoring for more than 6 charges (although none had gone to court), stranger victims, unrelated victims, being less than 25 years old and never having a relationship. It does seem prejudicial to score the latter item since so few men with IDD have had relationships, but even without this item he would have been placed in the high-risk category. We also completed the Violence Risk Appraisal Guide (VRAG; Harris et al 2014) to assess his static risk for future violent assaults. His score of 4 placed him in category 5 which, despite the fact that he had never been violent, represents a medium risk for future violence. The factors making up this result were his young age, being single, some difficult behaviours with schooling and we counted failure to adhere to the conditions (warnings) following each apprehension by the police. Because of this assessment, we stated that while he was at a high risk for future sexual offences, he was not a high risk for violence and this represented the context for the Armidilo assessment.

On the Armidilo several risk factors were assessed as high (Table 1) including compliance with supervision, offence management, high sexual preoccupation and deviance (in relation to indecent exposure), emotional coping, impulse control and relationships. For the latter his parents were extremely defensive of his behaviour and tended to blame the females, the police and social workers involved in his case. However, set against these were strong protective factors when he lived in the residential setting. These included good staff training, good staff attitudes and knowledge of the case following the first 3 weeks, excellent staff consistency of management, and no staff or environmental changes. For the offender items Mr B had excellent compliance with supervision and treatment, some emerging insight into his offence management, some emerging understanding of the importance of impulse control and how to do this, no mental health or substance use issues, and good relationships with staff. He reported that he masturbated around 6 times a day and he was assessed for and 
commenced on anti-libidinal medication. At the time of the report on the risk assessment it had only just begun and so while it was not rated as a protective factor, a partial protection was posited in the assessment report. The "offender changes" items were not relevant since there had been insufficient time to evaluate any changes.

This assessment was persuasive and it was agreed at a reconvened multi agency conference that $\mathrm{Mr} \mathrm{B}$ could continue to live in the community residential home for a further 3 months with constant evaluation of progress. It was acknowledged within the conference that there had probably been countless incidents of indecent exposure over the years and the apparent increase following admission were due to increased observation facilitating detection of the act. In fact it was the protective factors that ameliorated the risk probability when considering Mr B's future. The outcome of the evaluation of protective factors was that he continued to comply with supervision, maintained treatment, and commenced on antilibidinal medication which produced a significant reduction in reported frequency of masturbation. His impulse control also improved noticeably to the extent that his father commented repeatedly on how enjoyable it now was to be with him when they were out. $\mathrm{Mr}$ B continued to live in the residential home and there were two reported incidents of indecent exposure over the following 12 months both in the first 3 months. While two incidents represent recidivism, it is a massive reduction in frequency of incidents when placed against previous behaviour.

Case 3: Mr C. aged late twenties, WAIS IQ 60.

A discharge was proposed for $\mathrm{Mr} \mathrm{C}$ from a group residential home to a single tenancy with 24-hour support. He had been charged and convicted some 5 years previously with 4 incidents of indecent assault against pre-pubescent boys. The proposed service was in another area and the psychologist completed a risk assessment which placed him in the very high-risk category for future offences. As a result, the service was reluctant to accept $\mathrm{Mr} \mathrm{C}$ for the 
tenancy. Our assessment followed the pattern described previously resulting in a Static 99 in the high-risk category and a VRAG suggesting low to medium risk for violence. In fact, $\mathrm{Mr} \mathrm{C}$ had never been violent. Following extensive treatment and supervision during the placement he was now showing few stable or acute risk factors on the Armidilo (table 1) and the proposed placement had strong protective factors in place with good consistent supervision, good compliance with supervision, no mental health or substance use issues, and no envisaged significant changes following any move. Although there were no signs of high sexual drive the Armidilo was rated conservatively to indicate that the proposed changes might have an unsettling effect on his sexuality. The protective factors assessment carried the discussion and $\mathrm{Mr} \mathrm{C}$ moved to the tenancy. Four years on from the move there have been no further incidents.

Case 4: Mr D. aged early fifties, IQ 58

Mr D is presented because he represents a failure of recommendations based on the protective factors of the Armidilo. He was referred for admission to a private residential home for people with IDD and was assessed in the manner described above. He had committed one sexual offence against a 14-year-old female some 20 years ago and then another 6 years ago. Neither were penetrative but on both occasions, he had used force. On both occasions he had been drinking alcohol to excess. He had been imprisoned for the latter offence. He had spent 4 years on a mixed hospital ward and was given free access to the grounds. He was completely compliant with his regime, showed little interest in other residents and none in the females even although, over the years, some had been of a young age (17 to 25). On the static 99 he was assessed as medium to high risk from relevant variables. There were almost no risk factors on the Armidilo assessment, beyond having constant access to victims in the hospital. He had shown no interest in alcohol at any time in the 4 years of admission but, cautiously, this was scored as a risk. The protective factors in 
the proposed placement were stronger than in his current hospital placement with closer, more consistent supervision and also supervision when out in the community. He showed no problems with impulse control and no evidence of sexual preoccupation. The assessor cautiously assumed some continued deviant sexual interest in teenage girls, although there was no evidence of it.

Despite there being general agreement that the placement was appropriate and that the protective factors were an effective counter to any risk, there were concerns raised by management advising of the opinion that the risk for the other residents and the organisation was too great. Management argued that there were female residents who would be at risk of assault if Mr D was admitted. This argument carried the discussion despite the evidence from the referring organisation and from the risk assessment.

\section{Short Discussion}

It is necessary to advocate a careful approach when reading case studies, because the qualitative information presented could be skewed by both the authors' experience and the individuals presented for illustration. In this study, the authors have made every attempt to be objective, presenting cases which show what is possible with the use of the protective scale of the Armidilo rather than have any attempt at representative cases. In fact, the cases are very real despite anonymization and the outcomes are exactly as happened but they may not be representative. Both Lindsay et al (2002) and Willis and Grace (2008) found that planned discharge arrangements, social supports and clinical follow up were protective factors in the risk for future incidents in offenders. The cases in this study give substance to such targeted planning and support in individual cases.

The study presents cases illustrating the use of the protective scale of the Armidilo risk assessment and the cases have been discussed as they have been presented. In three of the cases the argument was carried by strong protective factors and in the fourth, protective 
factors made no difference to the outcome. In the first two cases it seemed to the authors that the course of the person's life was influenced positively by the assessment which employed the protective scale of the Armidilo. It is important to stress that the assessments outlined, in a cautious fashion, the static and dynamic risks that were present for that individual. There was no attempt to minimise the risks that might be present. In fact, any possible risk factor was taken into account even if it was not indicated by behaviour in the last 2 or 3 years.

It is the case that professionals and agencies are, rightly, concerned when they hear about possible admission for sex offenders. A sex offence on the premises will be drastic for the victim concerned. There are also worries about others' perception of the agency that may have allowed such behaviour to occur. There is caution to the extent of avoidance when someone with a sex offence history is considered for acceptance to any service. The authors are knowledgeable and experienced within clinical practice relative to the reactions of the public when they become aware that a sex offender might be visiting or residing in nearby premises. It is, therefore, all the more important to conduct careful risk assessments with corresponding recommendations that are as realistic and accurate as possible. In the most wide ranging prediction study ever conducted, Tetlock and colleagues (Tetlock \& Mellers 2002; Mellers, et al 2014, 2015) found that the most accurate predictions involved gathering "an outside view", downplaying uniqueness, determined by the general likelihood of certain events happening given the circumstances and characteristics of the type of case being considered; followed by “an inside view”, emphasising uniqueness, determined by the specific considerations for this individual case. The "inside view" will change as the circumstances change for the event under consideration. The Armidilo procedure does precisely this with the Static 99 providing "an outside view" and the Armidilo providing the "inside view", allowing for the most accurate risk assessment predictions. 
The cases described do not illustrate all the potential scenarios for the conduct of a risk assessment. The most obvious is where the accepting accommodation has too few protective factors. Let us assume in the case of Mr B. his family wished to take him home because he had improved so much (in fact this had been proposed from time to time by the family). In this case the assessment might have included a much lower rate of supervision, poorer compliance with observation because of familiarity with parents, a lower compliance with medication, irregular attendance at treatment and poorer impulse control. All of this would have raised considerably the chances of reoffending through a lowering of the protective factors. In this way, it can be noted that the Armidilo can inform on positive and negative outcomes. In conclusion we would recommend that protective factors are included in all risk assessments.

\section{References.}


References

Abidin, Z., Davoren, M., Naughton, L., Gibbons, O., Nulty, A., \& Kennedy, H. G. (2013). Susceptibility (risk and protective) factors for in-patient violence and self-harm: Prospective study of structured professional judgement instruments START and SAPROF, DUNDRUM-3 and DUNDRUM-4 in forensic mental health services. $B M C$ Psychiatry, 13, Article 197. doi:10.1186/1471-244X-13-197

Blacker, J., Beech, A. R., Wilcox, D. T., \& Boer, D. P. (2011). The assessment of dynamic risk and recidivism in a sample of special needs sexual offenders. Psychology, Crime \& Law, 17(1), 75-92. doi:10.1080/10683160903392376

Boer, Haaven, Lambrick, Lindsay, McVilly, Sakdalan \& Frize (2013) ARMIDILO: the Assessment of Risk and Manageability of Individuals with Developmental and Intellectual Limitations who Offend. www.Armidilo.net

Boer, D. P., Tough, S., \& Haaven, J. (2004). Assessment of risk manageability of intellectually disabled sex offenders. Journal of Applied Research in Intellectual Disabilities, 17(4), 275-283. doi:10.1111/j.1468-3148.2004.00214.x

Boer D. P., McVilly K. R. \& Lambick F. (2007) Contextualising risk in the assessment of intellectually disabled individuals. Sexual Offender Treatment 2. Available from http://www.sexual-offender-treatment.org/index.php?id=59\&type=123

De Vogel, V., de Ruiter, C., Bouman, Y., \& de Vries Robbé, M. (2012). SAPROF. Guidelines for the assessment of protective factors for violence risk (2nd ed.). Utrecht: De Forensische Zorgspecialisten

de Vries Robbé, M., de Vogel, V., \& de Spa, E. (2011). Protective factors for violence risk in forensic psychiatric patients: A retrospective validation study of the SAPROF. International Journal of Forensic Mental Health, 10, 178-186. doi:10.1080/14999013.2011.600232 
de Vries Robbé, M., Mann, R., Maruna S. \& Thornton, D. (2015) An exploration of protective factors supporting desistance from sexual offending. Sexual Abuse: a Journal of Research and Treatment, 27, 15-33. doi: 10.1177/1079063214547582

Hanson, R.K., \& Thornton, D. (1999). Static-99: Improving actuarial risk assessments for sex offenders. (User report 1999-02). Ottawa: Department of the Solicitor General of Canada

Lindsay, W. R., Elliot, S. F., \& Astell, A. (2004a). Predictors of sexual offence recidivism in offenders with intellectual disabilities. Journal of Applied Research in Intellectual Disabilities, 17(4), 299-305. doi:10.1111/j.1468-3148.2004.00217.x

Lindsay, W.R., Finlay, C., Steptoe, L., Haut, F \& Brewster, E. (2017) Predictive validity of the dynamic risk assessment and management system (DRAMS) in individuals with intellectual disability residing in the community. Psychology, Crime and Law, in press Lindsay, W. R., Hogue, T. E., Taylor, J. L., Steptoe, L., Mooney, P., O'Brien, G., . . Smith, A. H. W. (2008). Risk assessment in offenders with intellectual disability. International Journal of Offender Therapy and Comparative Criminology, 52(1) doi:10.1177/0306624X07308111

Lindsay, W. R., Murphy, L., Smith, G., Murphy, D., Edwards, Z., Chittock, C., . . Young, S. J. (2004b). The dynamic risk assessment and management system: An assessment of immediate risk of violence for individuals with offending and challenging behaviour. Journal of Applied Research in Intellectual Disabilities, 17(4), 267-274. doi:10.1111/j.1468-3148.2004.00215.x

Lindsay, W.R., Smith, A. H. W., Law, J., Quinn, K., Anderson, A., Smith, A.... \& Allan. R. (2002) A Treatment service for sex offenders and abusers with intellectual disability: Characteristics of referrals and evaluation. Journal of Applied Research in Intellectual Disabilities, 15, 166-174 
Lofthouse, R. E., Lindsay, W. R., Totsika, V., Hastings, R. P., Boer, D. P., \& Haaven, J. L. (2013). Prospective dynamic assessment of risk of sexual reoffending in individuals with an intellectual disability and a history of sexual offending behaviour. Journal of Applied Research in Intellectual Disabilities, 26(5), 394-403. doi:10.1111/jar.12029

Lofthouse, R., Lindsay, W., Totsika, V., \& Hastings, R. (2012). How do static and dynamic risk factors work together to predict reoffending amongst offenders with ID? Journal of Intellectual Disability Research, 56(7-8), 683-683.

Mellers, B., Stone E, Murray, T Minster, A, Rohrbaugh, N, Bishop, M ....Tetlock, P. (2014) Identifying and cultivating superforecasters as a method of improving probabilistic predictions. Perspectives on Psychological Science, 10, 267-281

Mellers, B. A., Ungar, L., Baron, J., Ramos, J., Gurcay, B., Fincher, K., . . Tetlock, P. (2014). Psychological strategies for winning a geopolitical tournament. Psychological Science, 25, 1106-1115.

McMillan, D., Hastings, R. P., \& Coldwell, J. (2004). Clinical and actuarial prediction of physical violence in a forensic intellectual disability hospital: A longitudinal study. Journal of Applied Research in Intellectual Disabilities, 17(4), 255-265. doi:10.1111/j.1468-3148.2004.00213.x

Quinsey V. L. (2004) Risk assessment and management in community settings. In: Offenders with Developmental Disabilities (eds W. R. Lindsay, J. L. Taylor \& P. Sturmey), pp. 131-142.John Wiley \& Sons, Chichester, UK.

Quinsey, V. L., Book, A., \& Skilling, T. A. (2004). A follow-up of deinstitutionalized men with intellectual disabilities and histories of antisocial behaviour. Journal of Applied Research in Intellectual Disabilities, 17(4), 243-253. doi:10.1111/j.14683148.2004.00216.x 
Quinsey, V. L., Harris, G. T., Rice, M. E., \& Cormier, C. A. (1998). Violent offenders. Appraising and managing risk $\left(2^{\text {nd }}\right.$ ed $)$ Washington, DC: American Psychological Association.

Steptoe, L. R., Lindsay, W. R., Murphy, L., \& Young, S. J. (2008). Construct validity, reliability and predictive validity of the dynamic risk assessment and management system (DRAMS) in offenders with intellectual disability. Legal and Criminological Psychology, 13, 309-321. doi:10.1348/135532507X218251

Tetlock, P. E., \& Mellers, B. A. (2002). The great rationality debate. Psychological Science, 13, 94-99.

Webster, C. D., Martin, M., Brink, J., Nicholls, T. L., \& Desmarais, S. L. (2009). Short-Term Assessment of Risk and Treatability (START). Clinical guide for evaluation risk and recovery. Version 1.1. Ontario: St. Joseph's Healthcare Hamilton.

Willis, G. M., \& Grace, R. C. (2008). The quality of community reintegration planning for child molesters: Effects on sexual recidivism. Sexual Abuse: A Journal of Research and Treatment, 20, 218-240.

Wilson, C. M., Desmarais, S.L. , Nicholls T.L. \& Brink, J. (2010) The Role of Client Strengths in Assessments of Violence Risk Using the Short-Term Assessment of Risk and Treatability (START). International Journal of Forensic Mental Health, 9, 282293 doi.org/10.1080/14999013.2010.534694 
Table 1: the Armidilo assessment results for each of the four cases. R-risk factor rating from $0-2 ; \mathrm{P}=$ protective factor rating from $0-2$

\begin{tabular}{|c|c|c|c|c|c|c|c|c|}
\hline Armidilo item & Mr A & & Mr B & & $\mathrm{MrC}$ & & Mr D & \\
\hline Stable environment item & $\mathrm{R}$ & $\mathrm{P}$ & $\mathrm{R}$ & $\mathrm{P}$ & $\mathrm{R}$ & $\mathrm{P}$ & $\mathrm{R}$ & $\mathrm{P}$ \\
\hline Staff attitudes & 0 & 2 & 0 & 2 & 0 & 2 & 0 & 2 \\
\hline Staff communication & 0 & 2 & 0 & 2 & 0 & 2 & 0 & 2 \\
\hline Client knowledge & 0 & 2 & 0 & 2 & 0 & 2 & 0 & 2 \\
\hline Staff consistency & 0 & 2 & 0 & 2 & 0 & 2 & 0 & 2 \\
\hline \multicolumn{9}{|l|}{ Acute environment } \\
\hline Staff changes & 0 & 1 & 0 & 2 & 0 & 2 & 0 & 2 \\
\hline Monitoring changes & 0 & 2 & 0 & 2 & 0 & 2 & 0 & 2 \\
\hline Victim access & 1 & 1 & 1 & 2 & 1 & 2 & 2 & 2 \\
\hline Environment change & 0 & 0 & 0 & 2 & 0 & 2 & 0 & 2 \\
\hline \multicolumn{9}{|l|}{ Stable offender } \\
\hline Compliance supervision & 0 & 2 & 2 & 2 & 0 & 2 & 0 & 2 \\
\hline Compliance treatment & 0 & 2 & 1 & 2 & 0 & 2 & 0 & 2 \\
\hline Offence management & 1 & 2 & 2 & 1 & 0 & 2 & 0 & 2 \\
\hline Sexual deviance & 1 & 2 & 2 & $0(1)$ & 1 & 2 & 1 & 2 \\
\hline Sexual preoccupation, & 1 & 0 & 2 & $0(1)$ & 1 & 2 & 0 & 2 \\
\hline Emotional coping, & 1 & 2 & 2 & 1 & 1 & 2 & 0 & 2 \\
\hline Mental health & 1 & 2 & 0 & 2 & 0 & 2 & 0 & 2 \\
\hline Substance abuse, & 1 & 2 & 0 & 2 & 0 & 2 & 2 & 2 \\
\hline impulse control & 0 & 2 & 2 & 1 & 0 & 2 & 0 & 2 \\
\hline Relationships & 0 & 2 & 2 & 2 & 0 & 2 & 0 & 2 \\
\hline \multicolumn{9}{|l|}{ Acute offender changes } \\
\hline supervision or treatment, & 0 & 2 & & & 0 & 2 & 0 & 2 \\
\hline sexual preoccupation, & 0 & 1 & & & 0 & 1 & 0 & 2 \\
\hline victim related behaviours & 0 & 1 & & & 0 & 1 & 0 & 2 \\
\hline Emotional coping. & 0 & 1 & & & 0 & 2 & 0 & 2 \\
\hline
\end{tabular}




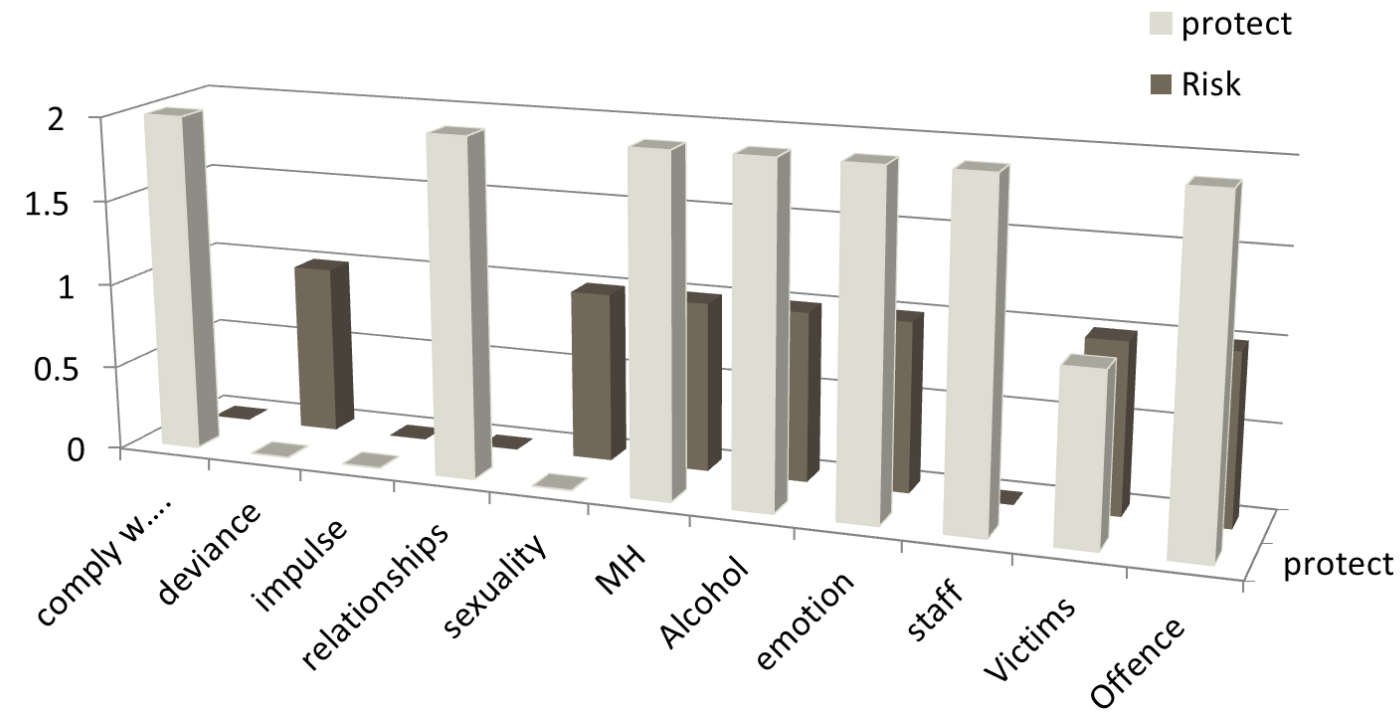

Figure 1. Mr A's risk and protective factors on the Armidilo assessment. 